\title{
Numerical resummation at subleading color in the strongly ordered soft gluon limit
}

\author{
Stefan Höche ${ }^{1}$ and Daniel Reichelt $\oplus^{2}$ \\ ${ }^{1}$ Fermi National Accelerator Laboratory, Batavia, Illinois 60510, USA \\ ${ }^{2}$ Institut für Theoretische Physik, Georg-August-Universität Göttingen, D-37077 Göttingen, Germany
}

(Received 3 February 2020; accepted 6 July 2021; published 9 August 2021)

\begin{abstract}
We present a Monte Carlo approach to soft-gluon resummation at subleading color which can be used to improve existing parton shower algorithms. At the single-emission level, soft-collinear enhancements of the splitting functions are explicitly linked to quadratic Casimir operators, while wide angle single-soft enhancements are connected to nontrivial color correlators. We focus on a numerically stable implementation of color matrix element corrections to all orders and approximate the virtual corrections by requiring unitarity at the single-emission level. We provide a proof-of-concept implementation to compute nonglobal event shapes at lepton colliders.
\end{abstract}

DOI: 10.1103/PhysRevD.104.034006

\section{INTRODUCTION}

Soft-gluon resummation is one of the most important tools in perturbative QCD, as it allows one to systematically and fairly straightforwardly compute radiative corrections to all orders for a large class of observables [1]. The effect of gluon radiation is typically computed for single or multiple emissions, and recoil effects are approximated at the same level. ${ }^{1}$ If the observable is simple, all-order corrections can be obtained by exponentiating these results, and the remaining obstacle is posed by color coherence, which may lead to a soft anomalous dimension in matrix form. A general framework for resumming event shapes based on this concept was developed at next-to-leading logarithmic (NLL) accuracy [3-6] and at next-to-next-toleading logarithmic accuracy $[7,8]$.

Nonglobal observables require a more sophisticated treatment, which was first discussed in the context of $e^{+} e^{-}$and deep inelastic scattering (DIS) event shape resummation $[9,10]$ using a Monte Carlo (MC) approach at leading color accuracy [9]. A corresponding evolution equation was derived [11], which enabled the inclusion of subleading color effects $[12,13]$. Numerical results have subsequently been computed for example for interjet energy flows [14] and for the hemisphere mass distribution in $e^{+} e^{-} \rightarrow$ hadrons

\footnotetext{
${ }^{1}$ This is known to cause potentially sizable differences between the results from parton showers and analytic resummation [2].

Published by the American Physical Society under the terms of the Creative Commons Attribution 4.0 International license. Further distribution of this work must maintain attribution to the author(s) and the published article's title, journal citation, and DOI. Funded by SCOAP ${ }^{3}$.
}

[15]. Nonglobal observables have also been investigated using methods of effective field theories [16-18].

Nonglobal logarithms are particularly important in the context of interjet radiation and in the presence of a jet veto $[19,20]$. In the context of Large Hadron Collider phenomenology they have therefore received considerable attention [21-25]. Several approaches have been suggested for their numerical resummation, ranging from subleading color parton showers [26-31] to evolution at the amplitude level [32-36]. While color-corrected parton showers can exhibit good numerical convergence, the evaluation of the color matrix elements becomes prohibitively expensive at high parton multiplicity, and therefore the approach cannot be used beyond a very limited number of emissions. Amplitudelevel evolution, on the other hand, will typically suffer from a slow rate of convergence in the Monte Carlo simulation. In order to address the problem of slow convergence, we propose a novel algorithm. Using color conservation, the squared soft-gluon current is rearranged into a soft-collinear contribution proportional to the quadratic Casimir operator and a collinearly suppressed correction term proportional to the color correlators. Based on the independence of color and kinematics operators, the color matrix elements are integrated with Monte Carlo methods at each step of the evolution. This allows one to reach good precision on the color coefficients, while limiting the run-time of computer simulations at high multiplicity. Appendix A shows how color coherence emerges in our approach.

This manuscript is organized as follows: Section II discusses the resummation formalism, and Sec. III introduces the phase-space mapping needed to implement it away from the exact soft limit. Section IV presents our Monte Carlo technique to compute the color matrix elements. The difference between subleading color and 
improved leading color evolution used in standard parton showers is analyzed in Sec. V by studying the light jet mass and narrow jet broadening distributions in $e^{+} e^{-} \rightarrow$ hadrons. Section VI contains an outlook.

\section{RESUMMATION FORMALISM}

Soft-gluon resummation is typically performed for a given, fixed number of hard partons, generated at scales that are widely separated from the scale of additional soft radiation. These partons are assumed to be unchanged after the emission of a soft gluon, leading to the notion of Wilson lines and eventually the exponentiation of the soft anomalous dimension matrix. We will adopt a different approach, based on the physical picture in the strongly ordered soft limit. By the very definition of strong ordering, each radiated soft gluon must be treated as a new Wilson line for subsequent gluon emissions.

We denote the Born matrix element for $n$ partons by $\left|M_{n}\right\rangle$ and the color insertion operator for parton $i$ as $\mathbf{T}_{i}[1]$. The approximate $n+1$-parton squared matrix element in the soft limit then reads

$$
\left\langle m_{n+1} \mid m_{n+1}\right\rangle=\left\langle M_{n}\left|\boldsymbol{\Gamma}_{n}(\mathbf{1})\right| M_{n}\right\rangle,
$$

where we have defined the squared $n$-parton soft current

$$
\boldsymbol{\Gamma}_{n}(\boldsymbol{\Gamma})=-\sum_{i=1}^{n} \sum_{\substack{j=1 \\ j \neq i}}^{n} \mathbf{T}_{i} \mathbf{\Gamma} \mathbf{T}_{j} w_{i j}, \quad \text { with } \quad w_{i j}=\frac{s_{i j}}{s_{i q} s_{j q}} .
$$

The invariants $s_{i j}$ are defined in terms of the parton momenta, $p_{i}$, as $s_{i j}=2 p_{i} p_{j}$. Since $\left|m_{n+1}\right\rangle$ is an $n+1$ parton matrix element, $\Gamma$ is defined in a higher dimensional color space than $\Gamma_{n}$. Equation (1) generalizes to $k+1$ emissions as

$$
\begin{aligned}
& \left\langle m_{n+k+1} \mid m_{n+k+1}\right\rangle \\
& =\left\langle m_{n+k}\left|\boldsymbol{\Gamma}_{n+k}(\mathbf{1})\right| m_{n+k}\right\rangle \\
& =\left\langle M_{n}\left|\boldsymbol{\Gamma}_{n}\left(\boldsymbol{\Gamma}_{n+1}\left(\cdots \boldsymbol{\Gamma}_{n+k-1}\left(\boldsymbol{\Gamma}_{n+k}(\mathbf{1})\right) \cdots\right)\right)\right| M_{n}\right\rangle .
\end{aligned}
$$

Note that the complexity of $\Gamma_{n}$ increases rapidly with the number of partons, such that the evaluation of color factors encoded in Eq. (3) becomes increasingly cumbersome. The evolution of the parton ensemble is governed by the differential branching probability

$$
\frac{\mathrm{d} \sigma_{n+k+1}}{\sigma_{n+k}}=\mathrm{d} \Phi_{+1} 8 \pi \alpha_{s} \frac{\left\langle m_{n+k}\left|\boldsymbol{\Gamma}_{n+k}(\mathbf{1})\right| m_{n+k}\right\rangle}{\left\langle m_{n+k} \mid m_{n+k}\right\rangle},
$$

where $\mathrm{d} \Phi_{+1}=\mathrm{d}^{4} q \delta\left(q^{2}\right) /(2 \pi)^{3}$ is the four-dimensional differential phase-space element for the emission of the gluon with momentum $q$. We parametrize this phase space as

$$
\mathrm{d} \Phi_{+1}=\frac{1}{16 \pi^{2}} \mathrm{~d} \kappa_{i j}^{2} \mathrm{~d} \tilde{z}_{i} \frac{\mathrm{d} \phi_{i j}}{2 \pi} J\left(\kappa_{i j}^{2}, \tilde{z}_{i}, \phi_{i j, m}\right),
$$

where $\kappa_{i j}^{2}$ is the evolution variable of the parton shower, $\tilde{z}_{i}$ is the splitting variable, $\phi_{i j}$ is an azimuthal angle, and $J\left(\kappa_{i j}^{2}, \tilde{z}_{i}, \phi_{i j, m}\right)$ is a Jacobian factor. For details, see Sec. III.

Equation (4) describes resolved real-emission corrections. A standard choice for parton-shower algorithms is to define a no-branching probability, $\Pi\left(\kappa^{2}\right)$, such that virtual and unresolved real-emission corrections are defined in terms of the resolved real-emission corrections by means of unitarity. This approach should be improved by accounting for the different color structure in virtual corrections [30,32,33], as well as for Coulomb phases [32,33,37]. It was pointed out in [38] that one can therefore not yet claim a fully color correct evolution. We will postpone these problems to a future publication. Instead we focus our attention on a suitable rearrangement of color and kinematics factors in the real components, in order to improve the numerical convergence of the simulation. While this is not sufficient for arbitrarily complicated observables, it constitutes an important step toward a complete full-color resummation algorithm.

We define the no-branching probability such as to restore unitarity

$$
\int_{t}^{Q^{2}} \mathrm{~d} \kappa_{i j}^{2} \frac{1}{\sigma_{n+k}} \int \frac{\mathrm{d} \sigma_{n+k+1}}{\mathrm{~d} \kappa_{i j}^{2}} \Pi\left(\kappa_{i j}^{2}, Q^{2}, \mathbf{1}\right)=1-\Pi\left(t, Q^{2}, \mathbf{1}\right) .
$$

This equation has the solution

$$
\Pi\left(t, Q^{2}, \boldsymbol{\Gamma}\right)=\prod_{i=1}^{n} \prod_{\substack{j=1 \\ j \neq i}}^{n} \Pi_{i j}\left(t, Q^{2}, \boldsymbol{\Gamma}\right)
$$

where

$$
\Pi_{i j}\left(t, Q^{2}, \boldsymbol{\Gamma}\right)=\exp \left\{-\int_{t}^{Q^{2}} \frac{\mathrm{d} \kappa_{i j}^{2}}{\kappa_{i j}^{2}} \int \mathrm{d} \tilde{z}_{i} \int \frac{\mathrm{d} \phi_{i j}}{2 \pi} J\left(\kappa_{i j}^{2}, \tilde{z}_{i}, \phi_{i j}\right) \frac{\alpha_{s}}{2 \pi} \frac{\left\langle m_{n+k}\left|\mathbf{T}_{i} \boldsymbol{\Gamma} \mathbf{T}_{j}\right| m_{n+k}\right\rangle}{\left\langle m_{n+k} \mid m_{n+k}\right\rangle}\right\} .
$$

The squared $n$-parton soft current, Eq. (2), has a form which is not particularly suitable for implementation in numerical simulations. We use the partial fractioning approach of [39] to rearrange it as 


$$
\boldsymbol{\Gamma}_{n}(\boldsymbol{\Gamma})=-\frac{1}{2} \sum_{i=1}^{n} \sum_{\substack{j=1 \\ j \neq i}}^{n} \mathbf{T}_{i} \boldsymbol{\Gamma} \mathbf{T}_{j}\left(P_{j}^{i}+P_{i}^{j}\right)=-\frac{1}{2} \sum_{i=1}^{n} \sum_{\substack{j=1 \\ j \neq i}}^{n}\left(\mathbf{T}_{i} \boldsymbol{\Gamma} \mathbf{T}_{j}+\mathbf{T}_{j} \boldsymbol{\Gamma} \mathbf{T}_{i}\right) P_{j}^{i}
$$

where we have defined the splitting operator

$$
P_{j}^{i}=\frac{1}{s_{i q}} \frac{2 s_{i j}}{s_{i q}+s_{j q}}
$$

Note that in general $\mathbf{T}_{i} \boldsymbol{\Gamma} \mathbf{T}_{j}$ will not equal $\mathbf{T}_{j} \boldsymbol{\Gamma} \mathbf{T}_{i}$; hence we cannot combine the two terms on the right-hand side of Eq. (9). We use color conservation to rewrite them as

$$
\boldsymbol{\Gamma}_{n}(\boldsymbol{\Gamma})=\frac{1}{n-1} \sum_{i=1}^{n} \sum_{\substack{j=1 \\ j \neq i}}^{n}\left(\mathbf{T}_{i} \boldsymbol{\Gamma} \mathbf{T}_{i} P_{j}^{i}+\frac{1}{2} \sum_{\substack{k=1 \\ k \neq i, j}}^{n}\left(\mathbf{T}_{i} \boldsymbol{\Gamma} \mathbf{T}_{k}+\mathbf{T}_{k} \boldsymbol{\Gamma} \mathbf{T}_{i}\right) P_{j}^{i}-\frac{n-2}{2}\left(\mathbf{T}_{i} \boldsymbol{\Gamma} \mathbf{T}_{j}+\mathbf{T}_{j} \boldsymbol{\Gamma} \mathbf{T}_{i}\right) P_{j}^{i}\right) .
$$

Combining the second and the last terms in parentheses, we obtain ${ }^{2}$

$$
\boldsymbol{\Gamma}_{n}(\boldsymbol{\Gamma})=\frac{1}{n-1} \sum_{i=1}^{n} \sum_{\substack{j=1 \\ j \neq i}}^{n}\left(\mathbf{T}_{i} \boldsymbol{\Gamma} \mathbf{T}_{i} P_{j}^{i}+\frac{1}{2} \sum_{\substack{k=1 \\ k \neq i, j}}^{n}\left(\mathbf{T}_{i} \boldsymbol{\Gamma} \mathbf{T}_{k}+\mathbf{T}_{k} \boldsymbol{\Gamma} \mathbf{T}_{i}\right) \tilde{P}_{j k}^{i}\right)
$$

where we have defined the splitting operator [40]

$$
\tilde{P}_{j k}^{i}=P_{j}^{i}-P_{k}^{i}
$$

Note that $\tilde{P}$ tends to zero in the $i q$-collinear limit. Equation (12) should therefore be viewed as a rearrangement of Eq. (9), where the collinearly enhanced terms are made explicit, and the remainder is singly soft enhanced only. While additional rearrangements would allow one to achieve a further kinematical suppression by combining multiple operators as $\tilde{P}_{j k}^{i}+\tilde{P}_{i l}^{j}+\tilde{P}_{l i}^{k}+\tilde{P}_{k j}^{l}$, such rearrangements will produce additional terms proportional to $\mathbf{T}_{i} \boldsymbol{\Gamma} \mathbf{T}_{i}$. We find Eq. (12) to be the most suitable form for a Monte Carlo implementation. Examples for its relation to analytically known soft insertion operators are given in Appendix B.

\section{KINEMATICS MAPPING}

In order to implement Eq. (12) in a numerical simulation, the operator $\tilde{P}_{j k}^{i}$ must be well defined. When evaluating the difference between $P_{k}^{i}$ and $P_{j}^{i}$, we assume either that the underlying Born configurations are identical in both terms or that their difference gives rise to subleading power corrections. Since the latter may be difficult to prove in the general case, we use a kinematics mapping, which ensures that the underlying Born state is the same for identical $i$ and $q$. Such a mapping is defined, for example in [41-46], and is schematically depicted in Fig. 1. Here and in the

\footnotetext{
${ }^{2}$ In the special case of $\boldsymbol{\Gamma}=\mathbf{1}$, i.e., at fixed order, we can simplify Eq. (12) using the identity $\mathbf{T}_{i}^{2}=C_{i}$. This relation was used to reformulate the two-loop soft function in [40].
}

following, a tilde denotes momenta before the emission of the soft gluon.

We define the variables in Eq. (5) as follows:

$$
\begin{aligned}
\kappa_{i j}^{2}= & \frac{s_{i q} s_{j q}}{s_{i j}}, \quad \tilde{z}_{i}=\frac{s_{i j}}{s_{i j}+s_{j q}}, \\
2 \cos \phi_{i j}= & \sqrt{\frac{s_{n i} s_{n q}}{s_{i q}}} \sqrt{\frac{s_{n j} s_{n m}}{s_{j m}}} \\
& \times\left(\frac{s_{i m}}{s_{n j} s_{n q}}+\frac{s_{j q}}{s_{n i} s_{n m}}-\frac{s_{i j}}{s_{n m} s_{n q}}-\frac{s_{m q}}{s_{n i} s_{n j}}\right),
\end{aligned}
$$

where the lightlike reference vector $n^{\mu}$ is defined in Eq. (15). The lightlike auxiliary vector $m^{\mu}$ is given by $m^{\mu}=$ $K-K^{2} /(2 K n) n$ and defines the reference axis for $\phi_{i j}$ in the transverse plane. Note that the inverse mapping leads to the same $n+k$-parton momentum configuration for any choice of $p_{j}^{\mu}$ and $m^{\mu}$, as long as $p_{i}^{\mu}$ and $q$ are identical. This is an important feature needed for the rearrangement of color insertion operators in Sec. II.

The longitudinal recoil generated in the emission of the soft gluon $q$ is absorbed by all partons except the emitter. Due to our choice of evolution and splitting variables, the mapping depends nontrivially on the azimuthal angle. In order to construct the momenta, we first define the lightlike vectors

$$
\begin{aligned}
l^{\mu} & =\frac{1+s_{j K} / \tilde{\gamma}}{1+s_{j K} / \gamma} \tilde{p}_{i q}^{\mu}, \\
n^{\mu} & =\frac{1}{1+s_{i q} / \gamma}\left(\tilde{K}^{\mu}+\tilde{p}_{j}^{\mu}-\frac{\left(\tilde{K}+\tilde{p}_{j}\right)^{2}}{\gamma} \tilde{p}_{i q}^{\mu}\right),
\end{aligned}
$$



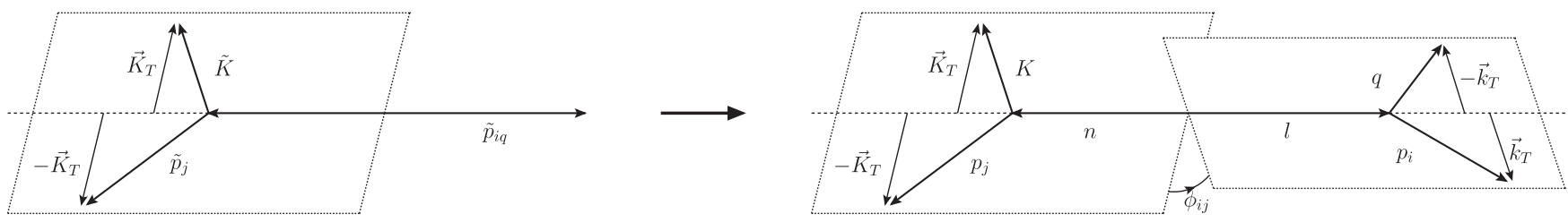

FIG. 1. Sketch of the kinematics mapping described in Sec. III. The emitting parton is $i$, and the reference momentum for definition of the azimuthal angle is $j$. The emitted gluon carries momentum $q$. The forward and backward light-cone momenta are given by $l$ and $n$.

where

$$
\tilde{\gamma}=2\left(\tilde{K}+\tilde{p}_{j}\right) \cdot \tilde{p}_{i q}=Q^{2}-\left(\tilde{K}+\tilde{p}_{j}\right)^{2}
$$

The rescaled invariant $\gamma$ is given by

$$
\gamma=\frac{1}{2}\left(\left(Q^{2}-\left(\tilde{K}+\tilde{p}_{j}\right)^{2}-s_{i q}\right)+\sqrt{\left(Q^{2}-\left(\tilde{K}+\tilde{p}_{j}\right)^{2}-s_{i q}\right)^{2}-4\left(\tilde{K}+\tilde{p}_{j}\right)^{2} s_{i q}}\right) .
$$

We can now parametrize the momenta as

$$
p_{i}^{\mu}=z l^{\mu}+\frac{\vec{k}_{T}^{2}}{z \gamma} n^{\mu}+k_{T}^{\mu}, \quad q^{\mu}=(1-z) l^{\mu}+\frac{\vec{k}_{T}^{2}}{(1-z) \gamma} n^{\mu}-k_{T}^{\mu}
$$

and

$$
p_{j}^{\mu}=x n^{\mu}+\frac{\vec{K}_{T}^{2}}{x \gamma} l^{\mu}-K_{T}^{\mu}, \quad K^{\mu}=(1-x) n^{\mu}+\frac{\tilde{K}^{2}+\vec{K}_{T}^{2}}{(1-x) \gamma} l^{\mu}+K_{T}^{\mu} .
$$

Note that $x$ and $K_{T}$ are invariant under the mapping, as the momenta $p_{j}$ and $K$ are completely determined by a boost of $\tilde{p}_{j}$ and $\tilde{K}$ along the direction of $n$. The variables $x$ and $\vec{K}_{T}^{2}$ can therefore be computed using the Born kinematics. Solving Eqs. (14) for $z$ and $\vec{k}_{T}^{2}$ then yields

$$
\begin{aligned}
z & =\frac{\tilde{z}_{i} C+\left(1-\tilde{z}_{i}\right) / C+2 \cos ^{2} \phi_{i j}-1}{(C-1)^{2} / C+4 \cos ^{2} \phi_{i j}}+\operatorname{sgn}\left(\cos \phi_{i j}\right) \frac{\sqrt{(C+1)^{2} / C \tilde{z}_{i}\left(1-\tilde{z}_{i}\right)-\sin ^{2} \phi_{i j}}}{(C-1)^{2} / C+4 \cos ^{2} \phi_{i j}}, \\
\vec{k}_{T}^{2} & =\kappa_{i j}^{2} \frac{\tilde{z}_{i}}{1-\tilde{z}_{i}} z(1-z), \quad \text { where } C=\frac{\left(1-\tilde{z}_{i}\right) x^{2}}{\tilde{z}_{i} \vec{K}_{T}^{2} \kappa_{i j}^{2}} .
\end{aligned}
$$

The phase-space boundaries are given by

$$
s_{i q} \leq Q^{2}-\tilde{K}^{2} \quad \text { and } \quad z<\left[1+\operatorname{sgn}\left(\cos \phi_{i j}\right) \sqrt{\vec{K}_{T}^{2} \kappa_{i j}^{2}} /(x \gamma)\right]^{-1} .
$$

The Jacobian introduced in Eq. (5) is given by

$$
J\left(\kappa_{i j}^{2}, \tilde{z}_{i}, \phi_{i j}\right)=\frac{\sqrt{\left(Q^{2}-\tilde{K}^{2}-s_{i q}\right)^{2}-4 \tilde{K}^{2} s_{i q}}}{Q^{2}-\tilde{K}^{2}} \frac{2 z(1-z)\left(z(1-z) x^{2} \gamma^{2}+\vec{K}_{T}^{2} \vec{k}_{T}^{2}\right)}{z(1-z) x \gamma\left(z s_{j q}+(1-z) s_{i j}\right)-\vec{K}_{T}^{2} \vec{k}_{T}^{2}} \frac{\kappa_{i j}^{2}}{\vec{k}_{T}^{2}}
$$

The fact that the inverse of this mapping yields the same underlying Born kinematics for all configurations where the emitting particle is parton $i$ allows one to rearrange the soft anomalous dimension matrix $\boldsymbol{\Gamma}$ into Eq. (12) without the need for an additional reweighting away from the exact soft limit. 


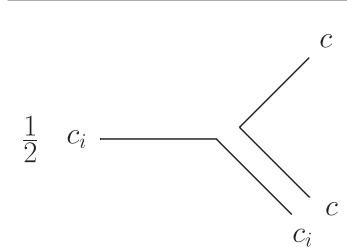

(a)

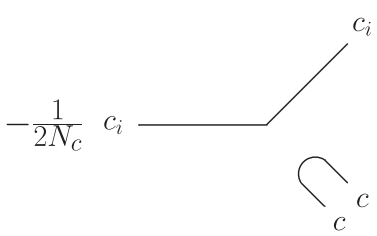

(b)

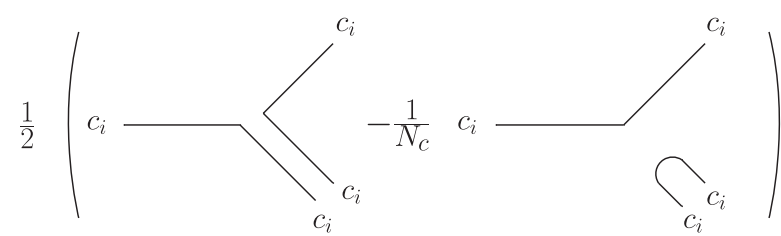

(c)

FIG. 2. Sketch of color flow in quark to quark gluon splittings. The original quark color state is $\left(c_{i}, 0\right)$. In case (a), the newly generated color $c$ differs from $c_{i}$, and the gluon is assigned the color state $\left(c_{i}, c\right)$. In case (b), the newly generated color $c$ differs from $c_{i}$, and the gluon is assigned the color state $(c, c)$. In case (c), the newly generated color is the same as the original color, $c_{i}$. The prefactors indicate the weight of each diagram, which originates in the Fierz identity $T_{i k}^{a} T_{m n}^{a}=1 / 2\left(\delta_{i n} \delta_{m k}-\delta_{i k} \delta_{m n} / N_{c}\right)$ and appears squared in the sampling algorithm. See the main text for details.

\section{COLOR ALGEBRA}

The color insertion operators $\mathbf{T}_{i} \cdots \mathbf{T}_{j}$ in Eq. (12) are computed using Monte Carlo summation in the colorflow basis. In the following, we denote the emission of a gluon off parton $i$ by the color branching $\left(c_{i}, \bar{c}_{i}\right) \rightarrow$ $\left(c_{i}^{\prime}, \bar{c}_{i}^{\prime}\right)\left(c_{g}^{\prime}, \bar{c}_{g}^{\prime}\right)$, and the absorption on parton $j$ by the color recombination $\left(c_{j}, \bar{c}_{j}\right)\left(c_{g}, \bar{c}_{g}\right) \rightarrow\left(c_{j}^{\prime}, \bar{c}_{j}^{\prime}\right)$. We choose to sample the color configuration in the emission according to the quadratic Casimir operator, $\mathbf{T}_{i}^{2}$. In the case of gluon emission off a quark, we have $\mathbf{T}_{i}^{2}=T_{i k}^{a} T_{k j}^{a}=C_{F} \delta_{i j}$. In order to allow for a fully differential sampling of the gluon color index, we insert a partition of unity in the form $\delta^{a b}=2 \operatorname{Tr}\left(T^{a} T^{b}\right)$, leading to $2\left(T_{i k}^{a} T_{m n}^{a}\right)\left(T_{n m}^{b} T_{k j}^{b}\right)$. The first parentheses are associated with the emission of the gluon, and the second with its absorption. With the help of the Fierz identity, $T_{i k}^{a} T_{m n}^{a}=1 / 2\left(\delta_{i n} \delta_{m k}-\delta_{i k} \delta_{m n} / N_{c}\right)$, we identify three distinct color topologies, which are depicted in Fig. 2. Diagram squared corresponds to contributions from $\delta_{i n} \delta_{m k}$ only, and carries a weight of $1 / 2$. For each given quark color $\left(c_{i}, 0\right)$, there are $N_{c}-1$ such configurations, leading to a relative weight of $\left(N_{c}-1\right) /\left(2 C_{F}\right)$. Diagram squared corresponds to contributions from $\delta_{i k} \delta_{m n}$ only, and carries a weight of $1 /\left(2 N_{c}^{2}\right)$. For each given quark color $\left(c_{i}, 0\right)$, there are $N_{c}-1$ such configurations, leading to a relative weight of $\left(N_{c}-1\right)\left(1 / N_{c}^{2}\right) /$ $\left(2 C_{F}\right)$. Diagram squared correspond to contributions from both $\delta_{\text {in }} \delta_{m k}$ and $\delta_{i k} \delta_{m n}$, and carry a weight of $\left(1-1 / N_{c}\right)^{2} / 2$. For each given quark color $\left(c_{i}, 0\right)$, there is exactly one such configuration, leading to a relative weight of $\left(1-1 / N_{c}\right)^{2} /\left(2 C_{F}\right)$.

Based on these considerations, and a similar decomposition of the quadratic Casimir operator for gluons, we are eventually led to the following color sampling algorithm:

1. If the emitter is a quark or an antiquark, assign a weight $C_{F}$ to the emission

(a) With probability $\left(N_{c}-1\right) /\left(2 C_{F}\right)$ generate an octet configuration:

if $i$ is a quark / antiquark, choose a new color $c \neq c_{i} / c \neq \bar{c}_{i}$

assign the flow as $\left(c_{i}, 0\right) \rightarrow(c, 0),\left(c_{i}, c\right) /$ $\left(0, c_{i}\right) \rightarrow(0, c),\left(c, c_{i}\right)$ (b) With probability $\left(N_{c}-1\right)\left(1 / N_{c}\right)^{2} /\left(2 C_{F}\right)$ generate a singlet configuration with different colors: if $i$ is a quark / antiquark, choose a new color $c \neq c_{i} / c \neq \bar{c}_{i}$,

set the color indices of the gluon to $(c, c)$

(c) With probability $\left(1-1 / N_{c}\right)^{2} /\left(2 C_{F}\right)$ generate a singlet configuration with identical colors:

if $i$ is a quark / antiquark,

set the color indices of the gluon to $\left(c_{i}, c_{i}\right) /\left(\bar{c}_{i}, \bar{c}_{i}\right)$

2. If the emitter is a gluon

(a) If $i$ is in an octet state, $c_{i} \neq \bar{c}_{i}$, choose a new color $c$ and assign the emission a weight $N_{c}$

(b) If $i$ is in a singlet state, $c_{i}=\bar{c}_{i}$, choose a new color $c \neq c_{i}$ and assign the emission a weight $N_{c}-1$

(c) Choose a random permutation, either $\left(c_{i, 1}, c_{i, 2}\right) \rightarrow$ $\left(c_{i, 1}, c\right),\left(c, c_{i, 2}\right)$ or $\left(c_{i, 1}, c_{i, 2}\right) \rightarrow\left(c, c_{i, 1}\right),\left(c_{i, 2}, c\right)$ The complete operator $\mathbf{T}_{i} \cdots \mathbf{T}_{j}$ is restored by sampling over all possible recombinations of the intermediate gluon upon insertion of $\mathbf{T}_{j}$. The recombination algorithm proceeds as follows:

1. If the absorber is a quark or antiquark

(a) If $j$ is a quark and $c_{j}=\bar{c}_{g}$ or $\bar{c}_{g}=c_{g}$, set the merged color to $\left(c_{g}, 0\right)$, else assign weight zero

(b) If $j$ is an antiquark and $\bar{c}_{j}=c_{g}$ or $c_{g}=\bar{c}_{g}$, set the merged color to $\left(0, \bar{c}_{g}\right)$, else assign weight zero

2. If the absorber is a gluon

(a) If $c_{g}=\bar{c}_{j}$ and $\bar{c}_{g}=c_{j}$, assign weight 2 and set the merged color randomly to either $\left(c_{j}, \bar{c}_{g}\right)$ or $\left(c_{g}, \bar{c}_{j}\right)$

(b) Else if $c_{g}=\bar{c}_{j} / \bar{c}_{g}=c_{j}$, set the merged color to $\left(c_{j}, \bar{c}_{g}\right) /\left(c_{g}, \bar{c}_{j}\right)$, else assign weight zero

Note that arbitrarily many insertions may happen before the gluon emitted by $\mathbf{T}_{i}$ is annihilated via $\mathbf{T}_{j}$, as required by Eq. (12). The correctness of the above algorithm follows directly from the decomposition of the generators and the structure constants of $S U\left(N_{c}\right)$ in the color-flow basis [47]. In the context of numerical resummation it is important to note that the color matrix elements in Eq. (8) can be evaluated as a Monte Carlo integral with more than one point per event. This can be 
TABLE I. Selected color coefficients of squared amplitudes in processes with two quarks at the leading order, normalized to the common overall factor of $N_{c}$. The colored lines in the diagrams represent gluons, while the black circle represents the quark loop. Numerical results have been obtained using the algorithm in Sec. IV and are given for $N_{c}=3$. Note that we define $F_{b c}^{a}=i f^{a b c}$.

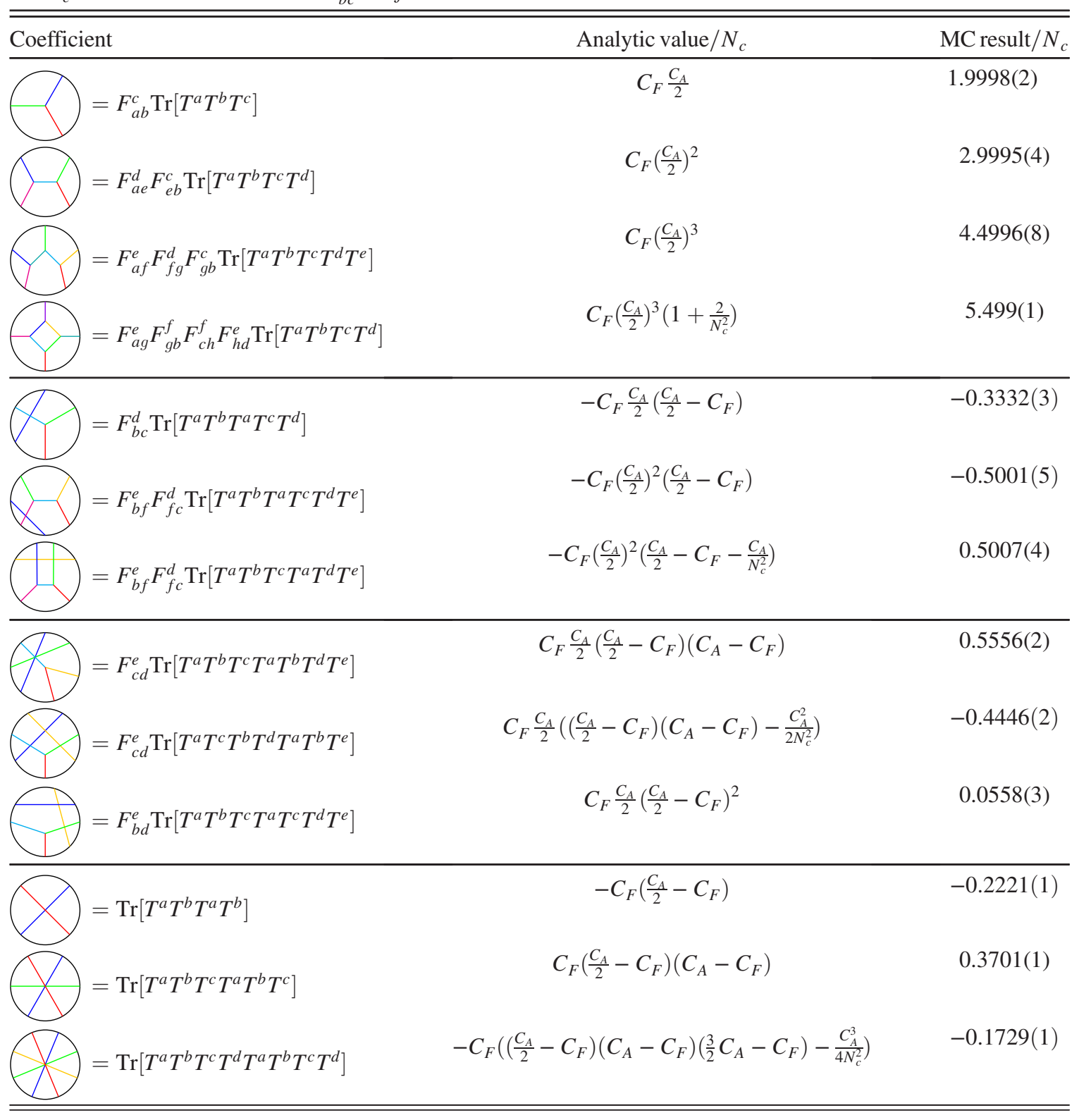

used in practice to improve the convergence of the overall simulation.

We validate the above algorithm numerically by computing the color coefficients for gluon webs within a quark loop. They can be systematically reduced to maximally non-Abelian coefficients, which are related to the quadratic, quartic, and higher-point Casimir operators. This leaves a small number of nontrivial intermediate gluon web configurations, which need to be evaluated. Table I lists some of these configurations up to four gluon insertions and compares the analytic results to Monte Carlo predictions from our algorithm at high statistical accuracy.
In this context we define $F_{b c}^{a}=i f^{a b c}$, where $f^{a b c}$ are the SU(3) structure constants. Note in particular that the fourth coefficient in the table is related to the quartic gluon Casimir operator, leading to an additional contribution of $2 / N_{c}^{2}$ which arises from double singlet gluon exchange between two gluons.

\section{NUMERICAL RESULTS}

In this section we apply our new algorithm to $e^{+} e^{-} \rightarrow$ jets at the $Z$ pole, $\sqrt{s}=91.2 \mathrm{GeV}$. We use a two-loop running coupling defined by $\alpha_{s}\left(Q^{2}\right)=0.118$, and the 

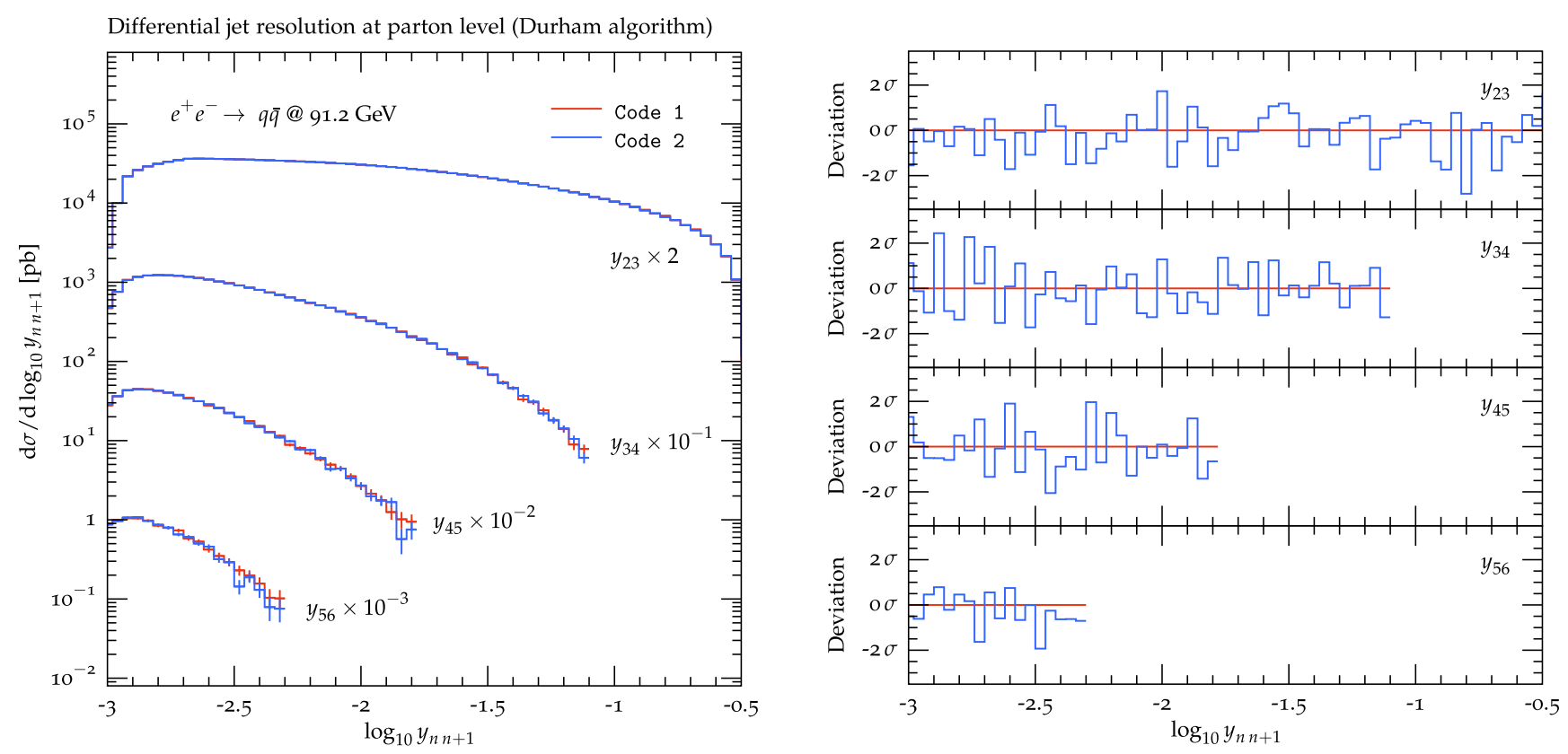

FIG. 3. Durham $k_{T}$-jet resolution scales [53] in $e^{+} e^{-} \rightarrow$ hadrons at $\sqrt{s}=91.2 \mathrm{GeV}$. We compare predictions from two independent implementations of our algorithm, labeled "Code 1" and "Code 2." The infrared cutoff is set to $\sqrt{t_{c}}=\sqrt{t_{c, F C}}=3 \mathrm{GeV}$.

quark mass thresholds $m_{c}=1.3 \mathrm{GeV}$ and $m_{b}=4.75 \mathrm{GeV}$. We cross-check our predictions using two entirely independent Monte Carlo implementations based on [48], which was validated independently against [49] at high precision. Our simulations do not include collinear contributions to the splitting functions, and they are carried out at the parton level. They can therefore not be compared directly to experimental data. However, they serve as a first proof-of-concept that color matrix element corrections at arbitrary multiplicity can be performed in a numerically stable fashion that enables their application to relevant physics problems in current or past collider experiments.

We follow the approach in [50] and terminate the subleading color evolution at a scale $t_{c, F C}$ that is insignificantly larger than the typical parton-shower infrared cutoff of $\sqrt{t_{c}} \sim 1 \mathrm{GeV}$. All distributions presented here are generated with $\sqrt{t_{c, F C}}=3 \mathrm{GeV}$. We claim that this is not a problem for practical applications, since hadronization effects typically influence numerical predictions up to a scale of the order of the $b$-quark mass, and the details of the fragmentation model have a much larger impact on measurable distributions in this range than the details of the parton shower. In order to provide a smooth transition to improved leading-color evolution below $t_{c, F C}$, we choose a leading color state according to the probability for a leading-color matrix element to have produced the partonic final state at scale $t_{c, F C}$. This is similar to how leading-color configurations are chosen in matching and merging techniques [51,52].

Figure 3 shows a comparison of predictions for the Durham $k_{T}$-jet rates [53] in $e^{+} e^{-} \rightarrow$ hadrons at $\sqrt{s}=$ $91.2 \mathrm{GeV}$. Our two independent numerical implementations of the resummation are statistically compatible and show good convergence, even in regions of large $k_{T}$ and for higher jet multiplicity. Figure 4 displays numerical predictions at $\sqrt{t_{c}}=1 \mathrm{GeV}$ for the Durham $2 \rightarrow 3$ and $3 \rightarrow 4$ jet scales, and for two nonglobal shape observables, the narrow jet broadening, $B_{N}$, and the light jet mass, $M_{L}$. We find that the impact of subleading color evolution on all these observables is less than $10 \%$, which agrees with the intuitive notion that corrections to improved leading color evolution should be of order $1 / N_{c}^{2}$. This can be taken as a strong indication that the typically excellent agreement of modern parton-shower predictions with measured nonglobal shape observables is not entirely accidental. A variant of the Durham $n \rightarrow(n+$ 1) jet scales has been resummed recently and matched to next-to-leading order (NLO), to achieve NLO + NLL' accuracy in [54], including a quantification of prospective subleading color contributions. Our results are compatible with the smallness of the effects observed there, in particular when noting that the results in [54] are matched to a fixed order NLO calculation relative to the Born process while we present pure parton shower results here. 

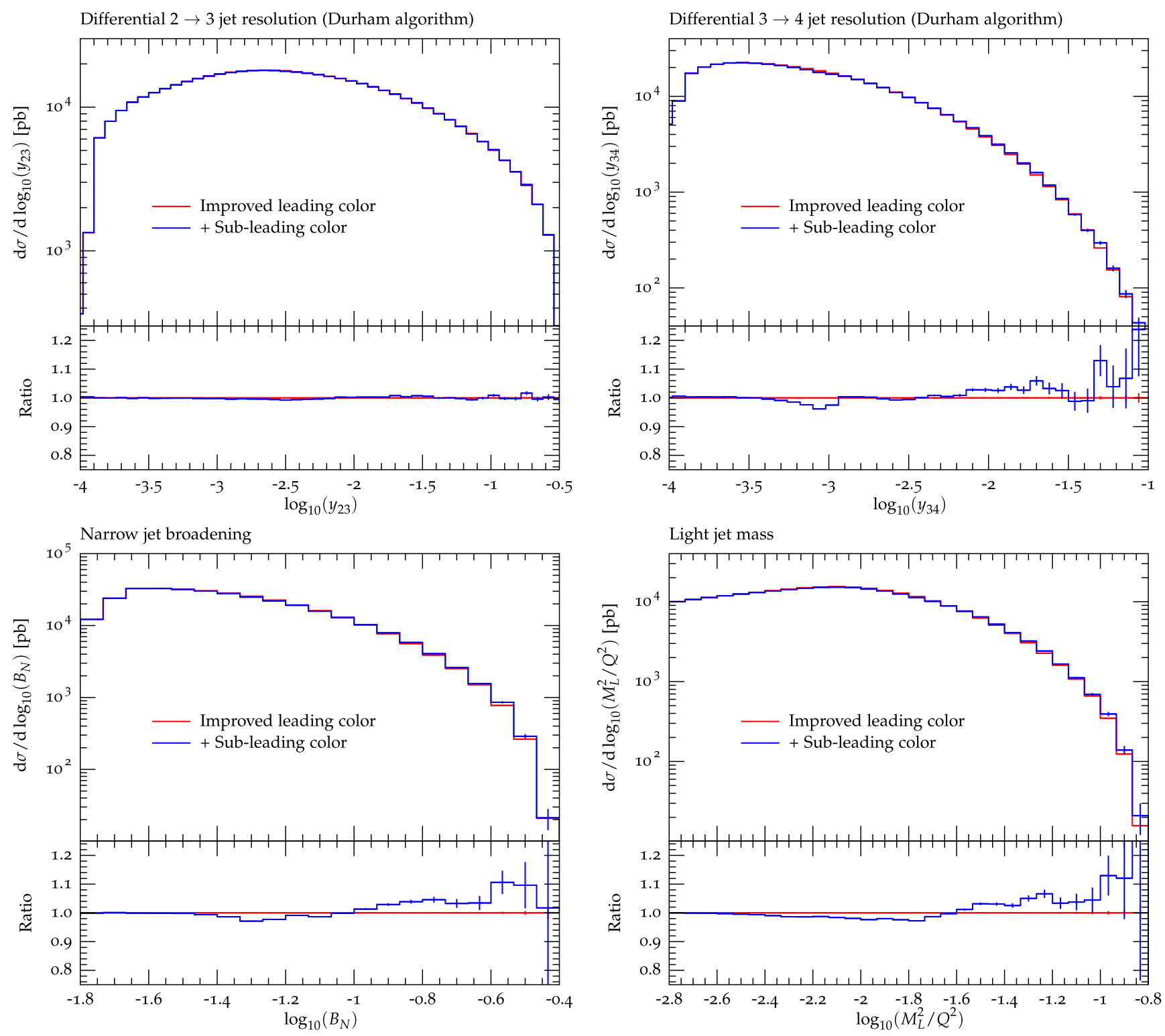

FIG. 4. Predictions for Durham $k_{T}$-jet resolution scales (top), narrow jet broadening (bottom left), and light jet mass (bottom right) in $e^{+} e^{-} \rightarrow$ hadrons at $\sqrt{s}=91.2 \mathrm{GeV}$. The infrared cutoffs are set to $\sqrt{t_{c}}=1 \mathrm{GeV}$ and $\sqrt{t_{c, F C}}=3 \mathrm{GeV}$. Results using an improved leading color approximation are shown in red, and results from the subleading color resummation are in blue.

\section{CONCLUSIONS}

We have presented a novel Monte Carlo method for softgluon resummation that allows one to generate parton-level events and can be incorporated into existing parton showers in order to improve their formal precision. Along with this manuscript, we provide a proof-of-concept implementation that can be used for numerical studies in $e^{+} e^{-} \rightarrow$ hadrons. We find that the impact of subleading color evolution on the Durham $k_{T}$-jet scales, on narrow jet broadening, and on the light jet mass agrees with the naive expectation that corrections to existing parton-shower approaches should be suppressed by $\mathcal{O}\left(1 / N_{c}^{2}\right)$. It will be interesting to investigate the impact on dedicated observables, which probe nontrivial color correlations, as for example in [55]. In this work we have neglected the matrix structure of soft virtual corrections. A future study will address the feasibility of including these contributions as well.

\section{ACKNOWLEDGMENTS}

We thank Joshua Isaacson for numerous fruitful discussions on the stochastic sampling of color configurations. We are grateful to Simone Marzani for his comments on the manuscript, and for many stimulating discussions on softgluon resummation. D. R. thanks Steffen Schumann for his support. We are particularly grateful to Dave Soper and Zoltan Nagy, as well as Jeff Forshaw, Simon Plätzer, 
and Jack Holguin for pointing out that our original claim to achieve resummation at full color accuracy does not hold due to the unitarity argument in Eq. (7) [38]. This work used resources of the Fermi National Accelerator Laboratory (Fermilab), a U.S. Department of Energy, Office of Science, HEP User Facility. Fermilab is managed by Fermi Research Alliance, LLC (FRA), acting under Contract No. DE-AC02-07CH11359. D. R. acknowledges support from the German-American Fulbright Commission which funded his research visit to Fermilab. D. R. further acknowledges funding from the European Unions Horizon 2020 research and innovation program as part of the Marie Skodowska-Curie Innovative Training Network MCnetITN3 (Grant Agreement No. 722104) and from BMBF (Contract No. 05H18MGCA1).

\section{APPENDIX A: RELATION TO ANALYTIC RESUMMATION OF GLOBAL EVENT SHAPES}

A general framework for resumming a large class of observables has been developed in the context of the program Caesar [3-6]. This formalism, in particular including the computation of color structures for in principle arbitrary multiplicities, was automated in [56] and recently applied to resummed calculations in $e^{+} e^{-} \rightarrow$ jets [54,57]. In this formalism, color coherence is an integral part in treating multiple real emissions. We therefore find it relevant to test how this is reproduced in our algorithm.

For two emissions at large rapidity difference, color coherence implies that $\boldsymbol{\Gamma}_{n+1}$ should reduce to $\boldsymbol{\Gamma}_{n}$. We are thus left to compute the following difference:

$$
\begin{aligned}
\Delta \boldsymbol{\Gamma}_{n+1}(\boldsymbol{\Gamma})= & \boldsymbol{\Gamma}_{n}\left(\boldsymbol{\Gamma}_{n+1}(\boldsymbol{\Gamma})\right)-\boldsymbol{\Gamma}_{n}\left(\boldsymbol{\Gamma}_{n}(\boldsymbol{\Gamma})\right) \\
= & -\sum_{i, k=1}^{n} \sum_{\substack{j=1 \\
j \neq i}}^{n} \mathbf{T}_{i}\left(\mathbf{T}_{n+1} \boldsymbol{\Gamma} \mathbf{T}_{k}+\mathbf{T}_{k} \boldsymbol{\Gamma} \mathbf{T}_{n+1}\right) \\
& \times \mathbf{T}_{j} w_{n+1 k} w_{i j} .
\end{aligned}
$$

We rewrite this as

$$
\Delta \boldsymbol{\Gamma}_{n+1}(\boldsymbol{\Gamma})=-\sum_{i=1}^{n} \sum_{\substack{j=1 \\ j \neq i}}^{n} \sum_{k=1}^{n} \sum_{\substack{l=1 \\ l \neq k}}^{n} \mathbf{T}_{i} \mathbf{T}_{k} \Delta_{n}^{(k l)}(\boldsymbol{\Gamma}) \mathbf{T}_{l} \mathbf{T}_{j} w_{k l} w_{i j},
$$

where

$$
\Delta_{n}^{(k l)}(\boldsymbol{\Gamma})=\frac{\mathbf{T}_{n+1} \boldsymbol{\Gamma} \mathbf{T}_{l}}{\mathbf{T}_{k} \boldsymbol{\Gamma} \mathbf{T}_{l}} X_{n+1 q}^{k l}+\frac{\mathbf{T}_{k} \boldsymbol{\Gamma} \mathbf{T}_{n+1}}{\mathbf{T}_{k} \boldsymbol{\Gamma} \mathbf{T}_{l}} X_{n+1 q}^{k l},
$$

and where we have defined the cross ratio

$$
X_{i j}^{k l}=\frac{w_{i k}}{w_{k l}}=\frac{s_{i k} s_{l j}}{s_{i j} s_{k l}} .
$$

Note that $X_{i j}^{k l}$ does not scale if any of the particles becomes soft. We can parametrize it in terms of the rapidity difference $\Delta \eta_{k l}^{(i j)}$ and azimuthal angle difference $\Delta \phi_{k l}^{(i j)}$ with respect to the light-cone directions defined by $p_{i}$ and $p_{j}$,

$$
\begin{aligned}
\cosh \Delta \eta_{k l}^{(i j)} & =\frac{s_{i k} s_{j l}+s_{i l} s_{j k}}{\sqrt{s_{i k} s_{j k} s_{i l} s_{j l}}}, \\
\cos \Delta \phi_{k l}^{(i j)} & =\frac{s_{i k} s_{j l}+s_{i l} s_{j k}-s_{i j} s_{k l}}{\sqrt{s_{i k} s_{j k} s_{i l} s_{j l}}},
\end{aligned}
$$

such that

$$
X_{i j}^{k l}=\frac{\cosh \Delta \eta_{k l}^{(i j)}-\sinh \Delta \eta_{k l}^{(i j)}}{\cosh \Delta \eta_{k l}^{(i j)}-\cos \Delta \phi_{k l}^{(i j)}}
$$

Averaging over the azimuthal angle gives

$$
\bar{X}_{i j}^{k l}=\frac{1}{2 \pi} \int_{0}^{2 \pi} \mathrm{d} \Delta \phi_{k l}^{(i j)} X_{i j}^{k l}=\operatorname{coth} \Delta \eta_{k l}^{(i j)}-1 .
$$

For the global, recursively infrared, and collinear safe observables considered in [3-6], the region $\Delta \eta_{i q} \ll 1$ is completely described by implementing the collinear evolution of gluon webs to the desired accuracy, which only depends on (the quadratic color Casimir operator of) the original hard leg. The remaining groups of gluons have $1 / \Delta \eta \approx \alpha_{s} \ln (1 / \epsilon) \ll 1$. In this case, $\bar{X}_{i j}^{k l}$ vanishes, and we obtain $\int \mathrm{d} \phi \Delta_{n+1}(\boldsymbol{\Gamma}) \rightarrow 0$. We hence correctly reproduce the picture of [6]: The radiation of an additional soft gluon can either be computed in the collinear limit or it can be described using the original soft anomalous dimension, ignoring the change in color flow arising from previous soft-gluon insertions. A similar description of this effect is obtained in the coherent branching formalism [58].

\section{APPENDIX B: EXPLICIT EXAMPLES OF INSERTION OPERATORS}

In this appendix we demonstrate the application of the soft-gluon insertion formula, Eq. (12), using two simple examples. The two-parton case being trivial, we investigate soft insertions into three- and four-parton matrix elements, as they occur, for example, in $e^{+} e^{-} \rightarrow$ hadrons or $h \rightarrow g g$ decays. Due to crossing invariance of the hard matrix elements, these examples also cover the highly relevant cases of charged and neutral current Drell-Yan and Higgsboson production at hadron colliders, as well as charged and neutral current deep inelastic scattering.

\section{Three radiators}

All elements of the color algebra can be expressed in terms of the quadratic Casimir operators $\mathbf{T}_{1}^{2}, \mathbf{T}_{2}^{2}$, and $\mathbf{T}_{3}^{2}$ by means of color charge conservation, $\mathbf{T}_{1}+\mathbf{T}_{2}+\mathbf{T}_{3}=0$. The remaining insertion operators can be written as 


$$
\begin{aligned}
& \mathbf{T}_{1} \mathbf{T}_{2}=\frac{1}{2}\left(-C_{1}-C_{2}+C_{3}\right), \\
& \mathbf{T}_{1} \mathbf{T}_{3}=\frac{1}{2}\left(-C_{1}+C_{2}-C_{3}\right), \\
& \mathbf{T}_{2} \mathbf{T}_{3}=\frac{1}{2}\left(C_{1}-C_{2}-C_{3}\right) .
\end{aligned}
$$

Based on Eq. (12), the complete soft insertion operator with 1,2 being the same type of parton (either quark or gluon) is then given by

$$
\begin{aligned}
\boldsymbol{\Gamma}_{3}(\mathbf{1})= & \frac{1}{2} \sum_{i=1}^{3} \sum_{\substack{j=1 \\
j \neq i}}^{3}\left(\mathbf{T}_{i}^{2} P_{j}^{i}+\sum_{\substack{k=j+1 \\
k \neq i, j}}^{3} \mathbf{T}_{i} \mathbf{T}_{j} \tilde{P}_{k j}^{i}\right) \\
= & \frac{1}{2}\left(C_{1}\left(P_{2}^{1}+P_{3}^{1}+P_{1}^{2}+P_{3}^{2}\right)+C_{3}\left(P_{1}^{3}+P_{2}^{3}\right)\right. \\
& \left.+\left(\frac{C_{3}}{2}-C_{1}\right)\left(\tilde{P}_{32}^{1}+\tilde{P}_{31}^{2}\right)-\frac{C_{3}}{2}\left(\tilde{P}_{23}^{1}+\tilde{P}_{13}^{2}\right)\right) \\
= & C_{1} w_{12}+\frac{C_{3}}{2}\left(w_{13}+w_{23}-w_{12}\right) .
\end{aligned}
$$

\section{Four radiators}

We choose $\mathbf{T}_{1}^{2}, \ldots, \mathbf{T}_{4}^{2}, \mathbf{T}_{1} \mathbf{T}_{4}$, and $\mathbf{T}_{1} \mathbf{T}_{3}$ to be the independent elements of the color algebra. The remaining insertion operators can be expressed in terms of these operators as

$\mathbf{T}_{1} \mathbf{T}_{2}=-C_{1}-\mathbf{T}_{1} \mathbf{T}_{3}-\mathbf{T}_{1} \mathbf{T}_{4}$

$\mathbf{T}_{2} \mathbf{T}_{3}=\frac{1}{2}\left(C_{1}-C_{2}-C_{3}+C_{4}\right)+\mathbf{T}_{1} \mathbf{T}_{4}$,

$\mathbf{T}_{2} \mathbf{T}_{4}=\frac{1}{2}\left(C_{1}-C_{2}+C_{3}-C_{4}\right)+\mathbf{T}_{1} \mathbf{T}_{3}$,

$\mathbf{T}_{3} \mathbf{T}_{4}=\frac{1}{2}\left(-C_{1}+C_{2}-C_{3}-C_{4}\right)-\mathbf{T}_{1} \mathbf{T}_{3}-\mathbf{T}_{1} \mathbf{T}_{4}$

Based on Eq. (12), the complete soft insertion operator for the four parton case with 1,2 and 3,4 being the same type of parton (either quark or gluon) then reads

$$
\begin{aligned}
\boldsymbol{\Gamma}_{4}(\mathbf{1})= & \frac{1}{3} \sum_{i=1}^{4} \sum_{\substack{j=1 \\
j \neq i}}^{4}\left(\mathbf{T}_{i}^{2} P_{j}^{i}+\sum_{\substack{k=i+1 \\
k \neq i, j}}^{4} \mathbf{T}_{i} \mathbf{T}_{j} \tilde{P}_{k j}^{i}\right) \\
= & \frac{1}{3}\left(C_{1}\left(P_{2}^{1}+P_{3}^{1}+P_{4}^{1}+P_{1}^{2}+P_{3}^{2}+P_{4}^{2}\right)+C_{3}\left(P_{1}^{3}+P_{2}^{3}+P_{4}^{3}+P_{1}^{4}+P_{2}^{4}+P_{3}^{4}\right)\right. \\
& +\mathbf{T}_{1} \mathbf{T}_{2}\left(\tilde{P}_{32}^{1}+\tilde{P}_{42}^{1}+\tilde{P}_{31}^{2}+\tilde{P}_{41}^{2}\right)+\mathbf{T}_{1} \mathbf{T}_{3}\left(\tilde{P}_{23}^{1}+\tilde{P}_{43}^{1}+\tilde{P}_{21}^{3}+\tilde{P}_{41}^{3}\right) \\
& +\mathbf{T}_{1} \mathbf{T}_{4}\left(\tilde{P}_{24}^{1}+\tilde{P}_{34}^{1}+\tilde{P}_{21}^{4}+\tilde{P}_{31}^{4}\right)+\mathbf{T}_{2} \mathbf{T}_{3}\left(\tilde{P}_{13}^{2}+\tilde{P}_{43}^{2}+\tilde{P}_{12}^{3}+\tilde{P}_{42}^{3}\right) \\
& \left.+\mathbf{T}_{2} \mathbf{T}_{4}\left(\tilde{P}_{14}^{2}+\tilde{P}_{34}^{2}+\tilde{P}_{12}^{4}+\tilde{P}_{32}^{4}\right)+\mathbf{T}_{3} \mathbf{T}_{4}\left(\tilde{P}_{14}^{3}+\tilde{P}_{24}^{3}+\tilde{P}_{13}^{4}+\tilde{P}_{23}^{4}\right)\right) \\
= & \frac{1}{3}\left(C_{1}\left(P_{2}^{1}+P_{3}^{1}+P_{4}^{1}+P_{1}^{2}+P_{3}^{2}+P_{4}^{2}\right)+C_{3}\left(P_{1}^{3}+P_{2}^{3}+P_{4}^{3}+P_{1}^{4}+P_{2}^{4}+P_{3}^{4}\right)\right. \\
& +3 \mathbf{T}_{1} \mathbf{T}_{3}\left(\tilde{P}_{23}^{1}+\tilde{P}_{14}^{2}+\tilde{P}_{41}^{3}+\tilde{P}_{32}^{4}\right)+3 \mathbf{T}_{1} \mathbf{T}_{4}\left(\tilde{P}_{24}^{1}+\tilde{P}_{13}^{2}+\tilde{P}_{42}^{3}+\tilde{P}_{31}^{4}\right) \\
& \left.-C_{1}\left(\tilde{P}_{32}^{1}+\tilde{P}_{42}^{1}+\tilde{P}_{31}^{2}+\tilde{P}_{41}^{2}\right)-C_{3}\left(\tilde{P}_{14}^{3}+\tilde{P}_{23}^{4}+\tilde{P}_{13}^{4}+\tilde{P}_{23}^{4}\right)\right) \\
= & C_{1} w_{12}+C_{3} w_{34}+\mathbf{T}_{1} \mathbf{T}_{3}\left(w_{12}+w_{34}-w_{13}-w_{24}\right)+\mathbf{T}_{1} \mathbf{T}_{4}\left(w_{12}+w_{34}-w_{14}-w_{23}\right) .
\end{aligned}
$$

[1] A. Bassetto, M. Ciafaloni, and G. Marchesini, Phys. Rep. 100, 201 (1983).

[2] S. Höche, D. Reichelt, and F. Siegert, J. High Energy Phys. 01 (2018) 118.

[3] A. Banfi, G. Salam, and G. Zanderighi, J. High Energy Phys. 01 (2002) 018.

[4] A. Banfi, G. P. Salam, and G. Zanderighi, Phys. Lett. B 584, 298 (2004).
[5] A. Banfi, G. P. Salam, and G. Zanderighi, J. High Energy Phys. 08 (2004) 062.

[6] A. Banfi, G. P. Salam, and G. Zanderighi, J. High Energy Phys. 03 (2005) 073.

[7] A. Banfi, H. McAslan, P. F. Monni, and G. Zanderighi, J. High Energy Phys. 05 (2015) 102.

[8] A. Banfi, H. McAslan, P. F. Monni, and G. Zanderighi, Phys. Rev. Lett. 117, 172001 (2016). 
[9] M. Dasgupta and G. Salam, Phys. Lett. B 512, 323 (2001).

[10] M. Dasgupta and G. P. Salam, J. High Energy Phys. 08 (2002) 032.

[11] A. Banfi, G. Marchesini, and G. Smye, J. High Energy Phys. 08 (2002) 006.

[12] H. Weigert, Nucl. Phys. B685, 321 (2004).

[13] S. Caron-Huot, J. High Energy Phys. 03 (2018) 036.

[14] Y. Hatta and T. Ueda, Nucl. Phys. B874, 808 (2013).

[15] Y. Hagiwara, Y. Hatta, and T. Ueda, Phys. Lett. B 756, 254 (2016).

[16] A. J. Larkoski, I. Moult, and D. Neill, J. High Energy Phys. 09 (2015) 143.

[17] T. Becher, M. Neubert, L. Rothen, and D. Y. Shao, Phys. Rev. Lett. 116, 192001 (2016).

[18] T. Becher, M. Neubert, L. Rothen, and D. Y. Shao, J. High Energy Phys. 11 (2016) 019; 05 (2017) 154(E).

[19] J. R. Forshaw, A. Kyrieleis, and M. Seymour, J. High Energy Phys. 08 (2006) 059.

[20] J. Forshaw, A. Kyrieleis, and M. Seymour, J. High Energy Phys. 09 (2008) 128.

[21] J. Forshaw, J. Keates, and S. Marzani, J. High Energy Phys. 07 (2009) 023.

[22] R. M. D. Delgado, J. R. Forshaw, S. Marzani, and M. H. Seymour, J. High Energy Phys. 08 (2011) 157.

[23] A. Banfi, G. P. Salam, and G. Zanderighi, J. High Energy Phys. 06 (2012) 159.

[24] A. Banfi, P. F. Monni, G. P. Salam, and G. Zanderighi, Phys. Rev. Lett. 109, 202001 (2012).

[25] A. Banfi, F. Caola, F. A. Dreyer, P. F. Monni, G. P. Salam, G. Zanderighi, and F. Dulat, J. High Energy Phys. 04 (2016) 049.

[26] G. Gustafson, Nucl. Phys. B392, 251 (1993).

[27] S. Plätzer and M. Sjödahl, J. High Energy Phys. 07 (2012) 042.

[28] S. Plätzer, M. Sjodahl, and J. Thorén, J. High Energy Phys. 11 (2018) 009.

[29] Z. Nagy and D. E. Soper, J. High Energy Phys. 06 (2012) 044.

[30] Z. Nagy and D. E. Soper, Phys. Rev. D 99, 054009 (2019).

[31] K. Hamilton, R. Medves, G. P. Salam, L. Scyboz, and G. Soyez, arXiv:2011.10054.

[32] R. Ángeles Martínez, M. De Angelis, J. R. Forshaw, S. Plätzer, and M. H. Seymour, J. High Energy Phys. 05 (2018) 044.

[33] J. R. Forshaw, J. Holguin, and S. Plätzer, J. High Energy Phys. 08 (2019) 145.
[34] M. De Angelis, J. R. Forshaw, and S. Plätzer, Phys. Rev. Lett. 126, 112001 (2021).

[35] J. Holguin, J. R. Forshaw, and S. Plätzer, Eur. Phys. J. C 81, 364 (2021).

[36] S. Plätzer and I. Ruffa, J. High Energy Phys. 06 (2021) 007.

[37] Z. Nagy and D. E. Soper, Phys. Rev. D 100, 074005 (2019).

[38] S. P. J. Holguin and J. R. Forshaw, arXiv:2003.06399.

[39] S. Catani and M. H. Seymour, Nucl. Phys. B485, 291 (1997).

[40] F. Dulat, S. Höche, and S. Prestel, Phys. Rev. D 98, 074013 (2018).

[41] Z. Nagy and D. E. Soper, J. High Energy Phys. 09 (2007) 114.

[42] Z. Nagy and D. E. Soper, J. High Energy Phys. 03 (2008) 030 .

[43] Z. Nagy and D. E. Soper, J. High Energy Phys. 07 (2008) 025.

[44] Z. Nagy and D. E. Soper, J. High Energy Phys. 06 (2014) 097.

[45] C. H. Chung, M. Kramer, and T. Robens, J. High Energy Phys. 06 (2011) 144.

[46] C.-H. Chung and T. Robens, Phys. Rev. D 87, 074032 (2013).

[47] F. Maltoni, K. Paul, T. Stelzer, and S. Willenbrock, Phys. Rev. D 67, 014026 (2003).

[48] S. Höche, in Proceedings of TASI 2014 (World Scientific, Singapore, 2015), pp. 235-295.

[49] S. Höche and S. Prestel, Eur. Phys. J. C 75, 461 (2015).

[50] J. Isaacson and S. Prestel, Phys. Rev. D 99, 014021 (2019).

[51] M. L. Mangano, M. Moretti, and R. Pittau, Nucl. Phys. B632, 343 (2002).

[52] S. Höche, F. Krauss, S. Schumann, and F. Siegert, J. High Energy Phys. 05 (2009) 053.

[53] S. Catani, Y. L. Dokshitzer, M. Olsson, G. Turnock, and B. R. Webber, Phys. Lett. B 269, 432 (1991).

[54] N. Baberuxki, C. T. Preuss, D. Reichelt, and S. Schumann, J. High Energy Phys. 04 (2020) 112.

[55] A. Larkoski, S. Marzani, and C. Wu, J. High Energy Phys. 01 (2020) 104.

[56] E. Gerwick, S. Höche, S. Marzani, and S. Schumann, J. High Energy Phys. 02 (2015) 106.

[57] S. Marzani, D. Reichelt, S. Schumann, G. Soyez, and V. Theeuwes, J. High Energy Phys. 11 (2019) 179.

[58] R. K. Ellis, W. J. Stirling, and B. R. Webber, $Q C D$ and Collider Physics, 1st ed., Cambridge Monographs on Particle Physics, Nuclear Physics and Cosmology Vol. 8 (Cambridge University Press, Cambridge, 1996), p. 435. 